\title{
Joint Reconstruction of Tracer Distribution and Background in Magnetic Particle Imaging
}

\author{
Marcel Straub ${ }^{\circledR}$ and Volkmar Schulz
}

\begin{abstract}
Magnetic particle imaging (MPI) is a novel tomographic imaging technique, which visualizes the distribution of a magnetic nanoparticle-based tracer material. However, reconstructed MPI images often suffer from an insufficiently compensated image background caused by rapid non-deterministic changes in the background signal of the imaging device. In particular, the signal-to-background ratio (SBR) of the images is reduced for lower tracer concentrations or longer acquisitions. The state-of-the-art procedure in MPI is to frequently measure the background signal during the sample measurement. Unfortunately, this requires a removal of the entire object from the scanner's field of view (FOV), which introduces dead time and repositioning artifacts. To overcome these considerable restrictions, we propose a novel method that uses two consecutive image acquisitions as input parameters for a simultaneous reconstruction of the tracer distribution, as well as the background signal. The two acquisitions differ by just a small spatial shift, while keeping the object always within the focus of a slightly reduced FOV. A linearly interpolated background between the initial and final background measurement is used to seed the iterative reconstruction. The method has been tested with simulations and phantom measurements. Overall, a substantial reduction of the image background was observed, and the image SBR is increased by a factor of 2(7) for the measurement (simulation) data.
\end{abstract}

Index Terms-Magnetic particle imaging (MPI), background signal, reconstruction, artifact suppression, least squares.

\section{INTRODUCTION}

$\mathbf{M}$ AGNETIC particle imaging (MPI) is a novel tracer based imaging technology [1], which quantitatively measures the concentration distribution of a tracer of superparamagnetic iron oxide nanoparticles (SPIO). Its fast volumetric in vivo imaging capability with 46 volumes per second at a physiological relevant tracer dosage makes MPI especially

Manuscript received October 27, 2017; accepted November 22, 2017. Date of publication January 26, 2018; date of current version May 1, 2018. This work was supported by the START-Program of the Faculty of Medicine, RWTH Aachen University. The work of M. Straub was supported by Philips. (Corresponding author: Marcel Straub.)

M. Straub is with the Department of Physics of Molecular Imaging Systems, Institute of Experimental Molecular Imaging, RWTH Aachen University, 52074 Aachen, Germany (e-mail: marcel.straub@ rwth-aachen.de).

V. Schulz is with the Department of Physics of Molecular Imaging Systems, Institute of Experimental Molecular Imaging, RWTH Aachen University, 52074 Aachen, Germany, and also with Oncology Solutions, Philips Research Europe, 5656 AE Eindhoven, The Netherlands (e-mail: schulz@pmi.rwth-aachen.de).

Color versions of one or more of the figures in this paper are available online at http://ieeexplore.ieee.org.

Digital Object Identifier 10.1109/TMI.2017.2777878 useful for dynamic studies, such as myocardial perfusion imaging [2]. Furthermore, MPI has been recently used in long-term cell tracking [3], [4], lung perfusion [5] and cancer detection [6] studies. Besides imaging, MPI has shown its capabilities for medical interventions by steering a magnetic catheter model [7].

The basic principle of MPI relies on the measurement of the non-linear response of the SPIOs to a harmonic alternating magnetic field. Spatial encoding is achieved by superimposing a static magnetic gradient field, which creates a field free point (FFP). Only particles in the vicinity of the FFP are excited by the alternating magnetic field. All particles outside of the FFP are saturated and do not contribute to the signal generation process. By superimposing three orthogonal homogeneous magnetic fields called drive fields, the FFP is moved in three dimensions through space. The drive fields are also used to excite the SPIOs. The change in magnetization of the SPIOs is measured with a receive coil. Besides the FFP approach, a magnetic gradient field featuring a field free line (FFL) [8], [9] can be used as well. This technique possibly increases signal to noise ratio (SNR) by up to an order of magnitude [10].

As other imaging modalities, MPI suffers from a background signal, which reduces the available dynamic range of the measurement [11]. The background is usually estimated by separate measurements before and/or after a sample was scanned. These standard background measurements require the absence of any tracer material from the FOV. Subsequently, the background signal is subtracted from the sample measurement signal. This method is sufficient if the background is almost constant and can be modeled by a piecewise linear interpolation over the sample measurement time. Published MPI images [12]-[14] show that this method is insufficient to fully remove the actual image domain background. For illustration, the temporal change of the background signal of our MPI device is shown in Fig. 1 at an exemplary frequency component. The impact on the reconstructed image is shown in Fig. 2. Since this frequency component is above the noise floor, it is essential for the image reconstruction. Nevertheless, large and rapid changes in the complex background are visible. In this case, where the background cannot be described by a simple model, it is necessary to measure the background more often. The time span between consecutive background measurements needs to be short enough to assume the background change between the measurements to be linear. As a background measurement requires the removal of the tracer material from the FOV, this introduces a dead time 


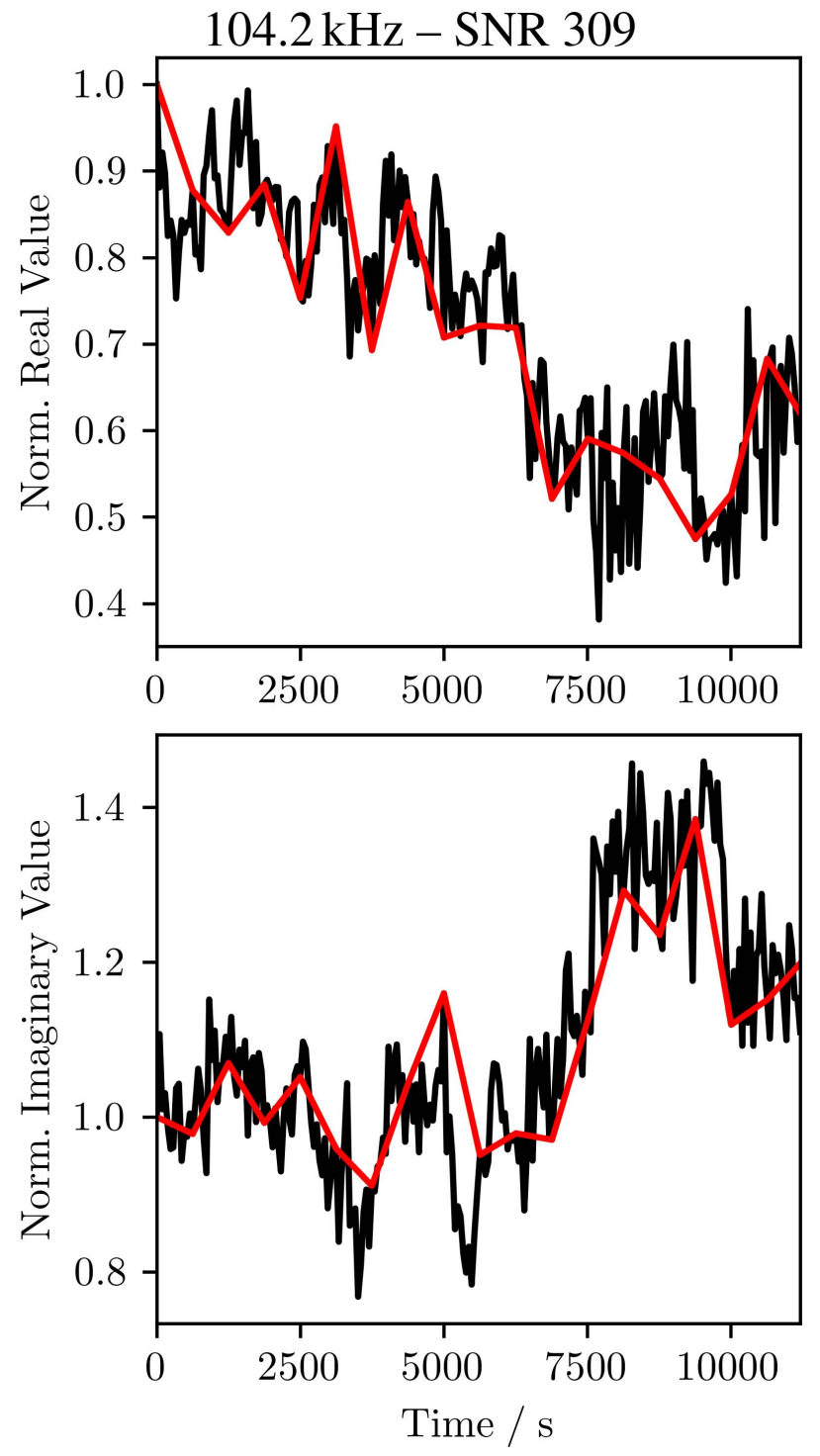

Fig. 1. Background signal at $104.168 \mathrm{kHz}$ of the $y$-axis receive channel over time (black). The background is measured every 19s. This frequency component is above the noise floor. The SNR is determined from a system matrix measurement with $0.8 \mu \mathrm{L}$ undiluted Resovist (see [19]). The signal is normalized to the first measured value. A piecewise linearly interpolated background in 10.4 min steps is shown in (red). Real (top) and imaginary (bottom) component of the complex signal.

as well as repositioning artifacts, which can be disadvantageous for dynamic studies. One approach to reduce the background originating from the drive field feedthrough signal is to use a gradiometer receive coil [15]-[17]. Furthermore, there exists a new reconstruction approach that aims at fitting the background during reconstruction [18]. This is achieved by extending the system matrix, which usually describes the system response to a delta probe of the tracer, by introducing a single additional "background-pixel" that holds a once measured background signal. Hence, during reconstruction the amplitude and phase of the "background-pixel" is fitted while the structure of the background is fixated.

In this work, we propose a new method that enables to extract the sample as well as the background signal simultaneously from two sample measurements. The basic idea is that due to the high temporal resolution of MPI, i.e. $22 \mathrm{~ms}$, the background as well as the tracer distribution can be assumed as quasi-static between two consecutive volumes. We exploit this by adding a well defined shift of the scanner FOV between two consecutive acquisitions of the quasi-static sample. So the actual signal of the sample changes between both acquisitions, while the background signal is kept static. The reconstruction formulation can be adapted to use this additional information to separate background and sample signal.

\section{METHOD}

\section{A. MPI Signal and Background}

The studies presented in this work are executed on the three-dimensional MPI scanner published in [2] which uses electronic FFP movement for tomographic image acquisition. Hence, this MPI device is equipped with three drive fields with frequencies of $24.5 \mathrm{kHz}, 26.0 \mathrm{kHz}$ and $25.3 \mathrm{kHz}$. It uses three orthogonal receive coils to measure magnetization changes in all three directions. The high sensitivity of the receive channels allows to measure the harmonic signal of the nonlinear particle response up to $1 \mathrm{MHz}$ (40th harmonic of the drive field signal). An 8th-order band-stop filter removes the direct feedthrough of the drive fields prior to amplification, preventing the saturation of the receive chain. The filtered and amplified signal is then discretized and digitized by a 12 bit ADC at $20 \mathrm{MSa} \mathrm{s}^{-1}$ (Adlink PCI 9812). After averaging, the effective bandwidth of the recorded signal is $2.5 \mathrm{MHz}$ per receive channel.

The digitized and discretized voltage signal $\tilde{\mathbf{u}} \in \mathbb{R}^{N_{\text {time }}}$ consists of $N_{\text {time }}$ time bins and is transformed into Fourier space

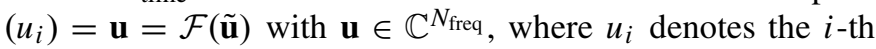
component of the vector and $N_{\text {freq }}$ the number of frequency bins. All frequency components that exhibit a low SNR, i.e. 10 here, are removed [19]. The removal of the noisy frequency components lowers the image noise and the amount of data by roughly $90 \%$. This results in reduced computation time for image reconstruction.

The frequency domain signal $\mathbf{u}$ can be divided into three components: the true particle signal $\mathbf{u}^{\text {true }} \in \mathbb{C}^{N_{\text {freq }}}$, the background signal $\mathbf{b} \in \mathbb{C}^{N_{\text {freq }}}$ and the Gaussian noise signal $\mathbf{n} \in \mathbb{C}^{N_{\text {freq }}}$. As these processes are independent, they superimpose to

$$
\mathbf{u}=\mathbf{u}^{\text {true }}+\mathbf{b}+\mathbf{n}
$$

The noise signal $\mathbf{n}$ can be reduced by averaging over multiple measurements and the background signal $\mathbf{b}$ is typically determined by an empty measurement, i.e. in absence of any tracer material. Hence, the true particle signal $\mathbf{u}^{\text {true }}$ can be theoretically extracted by subtracting the background component $\mathbf{b}$ and averaging over multiple measurements of the same sample to reduce the noise level. Unfortunately, the MPI background changes over time (see Fig. 1 and Fig. 2). Hence, this approach only works for a sufficiently small time difference between background and sample measurement.

\section{B. Reconstruction}

The MPI imaging process can be written as a forward projection of a discretized spatial tracer concentration distribution 


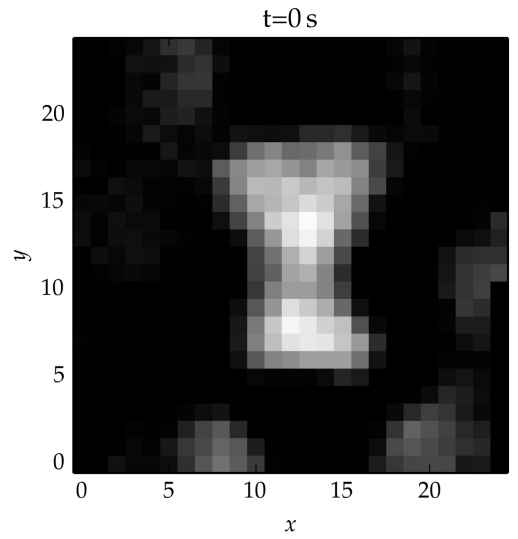

(a)

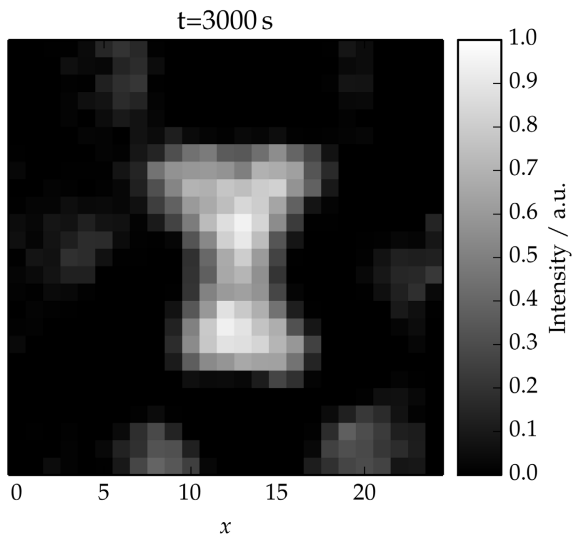

(b)

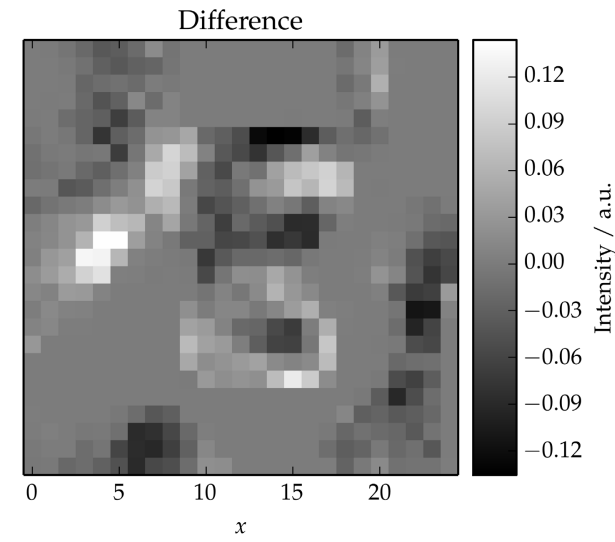

(c)

Fig. 2. MPI measurement of a statically positioned phantom filled with $32 \mu \mathrm{L}$ MM4 (Ferudextran, TOPASS GmbH, Berlin, Germany) at a concentration of $44 \mu \mathrm{gFe}_{\mathrm{Fe}} / \mathrm{mL}$ and measured over $55 \mathrm{~min}$. The measurements are reconstructed with the conventional Kaczmarz algorithm after background removal with a linearly interpolated background between a pre- and post background measurement. (a,b) Show the two reconstructed images recorded at the beginning and the end of the measurement utilizing the same gray scale for presentation. (c) Shows the difference between the two reconstructed images.

$\mathbf{c}(\mathbf{r}) \in \mathbb{C}^{N_{\text {pos }}}$ with $N_{\text {pos }}$ voxels to a measurement vector $\mathbf{u}$ via a transformation matrix $\mathbf{S} \in \mathbb{C}^{N_{\text {freq }} \times N_{\text {pos }}}[20]$

$$
\mathbf{S c}=\mathbf{u} .
$$

The tracer concentration distribution vector $\mathbf{c}$ can be bijectively mapped into an image matrix $\mathbf{x} \in \mathbb{R}^{N_{x} \times N_{y} \times N_{z}}$ with $N_{\text {pos }}=N_{x} \times N_{y} \times N_{z}$ voxels by a simple index mapping.

Since the transformation matrix describes the system's response to a particle distribution, it is called the system matrix. The system matrix can be determined either by measurement [19] or by simulation [21], [22]. A measurement based system matrix needs to be corrected for the background signal. This is usually achieved by interrupting the measurement every $20 \mathrm{~s}$ [14] to record the current background signal.

Hence, in MPI an image can be reconstructed from the measurement $\mathbf{u}$ by solving the inverse problem of (2), which is commonly done with a least squares fit [23]. For the system matrix based MPI reconstruction, it is common practice to apply the physical constraint that the concentration vector is positive and real during the reconstruction process [2], [24]. Introducing the weighting matrix $\mathbf{W} \in \mathbb{R}^{N_{\text {freq }}}$ and the $L_{2}$ regularization factor $\alpha \in \mathbb{R}^{\geq 0}$, the least squares formulation of the inverse problem is given by

$$
\|\mathbf{W}(\mathbf{S c}-\mathbf{u})\|_{2}^{2}+\alpha\|\mathbf{c}\|_{2}^{2} \rightarrow \min .
$$

The term $\alpha\|\mathbf{c}\|_{2}^{2}$ is used to compensate for the ill-conditioned system matrix $\mathbf{S}$ in (3). The weighting matrix $\mathbf{W}$ is used to weight the frequency components by e.g. their SNR, which can enhance the resulting image quality. In MPI, the iterative Kaczmarz method with non-negativity constraint [25] is widely used for solving (3). It converges rapidly due to the orthogonality of the system matrix [24] and results in a reasonable image quality.

Fig. 2a and Fig. 2b show the reconstructed images of the measurement of a phantom (see Fig. 5c) filled with $32 \mu \mathrm{L}$ MM4 (Ferudextran, TOPASS GmbH, Berlin, Germany) at a concentration of $44 \mu \mathrm{g}_{\mathrm{Fe}} / \mathrm{mL}$. The reconstructed images were recorded $3000 \mathrm{~s}$ apart from each other. Here, a slight change of the background is visible (see Fig. 2c). The measurements were background corrected with the conventional method and reconstructed with the non-negativity constraint Kaczmarz algorithm [25]. However, even though a background signal correction has been used, an image background is still visible in the reconstructed images.

Candidates for the source of the background signal are thermal processes in the scanner electronics, e.g. amplifiers, filters, coils and electrical contacts [11], as well as contamination with ferromagnetic materials and insufficient shielding. To reduce the influence of this slowly changing background, it is necessary to continuously measure the background signal. For this purpose, frequent background measurements without sample have to be performed in short intervals, e.g. every 20s [14], which are used for background interpolation. However, faster changes of the background are not covered well by this method as the linear interpolations usually fail. Most notably, the full removal of the sample is not a viable option for all experiments as it may introduce repositioning artifacts and substantial dead time, which is critical for dynamic studies.

\section{Joint Tracer and Background Reconstruction}

To avoid an interruption of the image acquisition, we propose to change the image acquisition scheme as well as the reconstruction process. We assume that the tracer distribution as well as the background do barely change between two consecutively recorded volumes. Due to the high temporal resolution of MPI, this seems to be a fair assumption. To separate the background and tracer signal, we introduce a well-known forward and backward shift of the scanner FOV between consecutive volumes (see Fig. 3). So besides noise only the particle but not the background signal has changed. The scanner FOV can be shifted between the consecutive images by superimposing a homogeneous magnetic field, e.g. with the so called focus field [26], or moving the sample physically with a robot. 


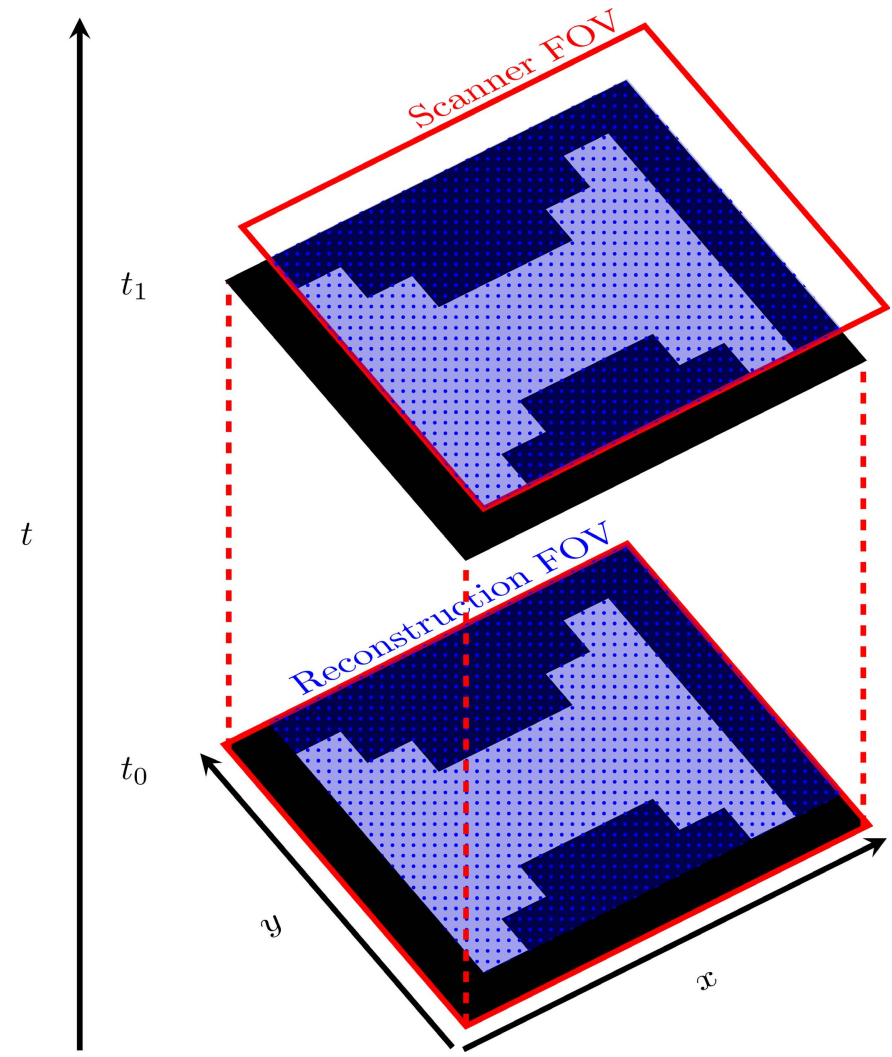

Fig. 3. Illustration of the joint reconstruction method for a twodimensional acquisition. Vertically, the time sequence of the image acquisition is shown. Here, the scanner FOVs (marked in red) at $t_{0}$ and $t_{1}$ are shifted against each other by one voxel in each spatial direction. The reconstruction FOV is a subset of the volume present in both scanner FOVs. It is the volume usable for the combined reconstruction and is marked by a blue overlay.

This results in two measurement vectors $\mathbf{u}_{0} \in \mathbb{C}^{N_{\text {freq }}}$ and $\mathbf{u}_{1} \in \mathbb{C}^{N_{\text {freq }}}$ for the unshifted $\left(t_{0}\right)$ and shifted $\left(t_{1}\right)$ scanner FOV, respectively. Both measurement vectors contain the true sample signal $\mathbf{u}_{i}^{\text {true }}$, an individual noise $\mathbf{n}_{i}$ component as well as a common background $\mathbf{b}$ :

$$
\mathbf{u}_{i}=\mathbf{u}_{i}^{\text {true }}+\mathbf{b}+\mathbf{n}_{i}
$$

For a short enough time span between both measurements (see Fig. 1), it can be justified to assume a common background signal.

With the knowledge that both image volumes just differ in a shift of the scanner FOV, which is denoted by the location vectors $\mathbf{r}_{i}$, we formulate a joint least squares reconstruction of the tracer concentration distribution $\mathbf{c}$ and the unknown background signal $\mathbf{b}$ from the independent measurements $\mathbf{u}_{i}$

$$
\begin{aligned}
\sum_{i=0}^{1} \| \mathbf{W}\left(\mathbf{S c}\left(\mathbf{r}_{i}\right)-\mathbf{u}_{i}\right. & +\mathbf{b}) \|_{2}^{2} \\
& +\alpha\|\mathbf{c}\|_{2}^{2}+\beta\left\|\mathbf{b}-\mathbf{b}_{\text {init }}\right\|_{2}^{2} \rightarrow \min .
\end{aligned}
$$

The system matrix $\mathbf{S}$ is already corrected for its background (see section II-B). As Fig. 1 suggests, this is required because the background usually differs between system matrix and sample measurement due the relative large temporal distance.
The reconstruction FOV (see Fig. 3) of the concentration vector $\mathbf{c}$ is a subset of the voxels present in the shifted and the unshifted scanner FOV. The term $\beta\left\|\mathbf{b}-\mathbf{b}_{\text {init }}\right\|_{2}^{2}$ with the parameter $\beta \in \mathbb{R}^{\geq 0}$ describes the background spectrum structure as a prior and is based on a previously measured or fitted background $\mathbf{b}_{\text {init }}$. Please note that $\mathbf{b}$ is in the signal domain. For small changes of the true tracer distribution and background, the resulting concentration distribution $\mathbf{c}$ as well as the estimated background $\mathbf{b}$ can be assumed to be averages from both measurements. This implies that the effective available image acquisition rate is reduced by a factor of two. In the following sections, we call this method the joint reconstruction.

To minimize (5) we use the iterative limited-memory Broyden-Fletcher-Goldfarb-Shanno algorithm with an extension to handle simple box constraints (L-BFGS-B) [27], [28]. The reduced memory usage enables its use on problems with a large number of variables, more than 1000, like it is the case for MPI image reconstruction. It is a quasi-Newton method that simultaneously optimizes $\mathbf{b}$ and $\mathbf{c}$. For the concentration vector c, a positivity constraint is applied as it is done for the Kaczmarz reconstruction [2], [25]. As the stopping criterion we choose the maximum norm of the projected gradient to be below $10^{-1}$. This reproducibly results in useful images of concentration distributions. We use the warmstarting method [29] that reduces the number of iterations by using an initial guess for the reconstruction result. Due to the high temporal resolution of MPI, a reasonable initial guess is the previously reconstructed volume. For the first volume of a sequence, the image reconstructed with the Kaczmarz algorithm of the very same measurement is reasonable.

\section{Simulation Studies}

In order to demonstrate the benefits of the proposed method, we performed simulation studies. For the simulations, we used a measured system matrix, a measured background and simulated Gaussian noise. Since we do not have a physical model describing the MPI background, we used a measured background series to model it as accurately as possible. We gained the background series from a measurement of about $20 \mathrm{~min}$. This measurement was interrupted every $19 \mathrm{~s}$ to acquire the background signal over $2 \mathrm{~s}$. Each background measurement was equally temporally divided in two groups: $A$ and $B$. So we denote the background signal measured at time point $j$ and assigned to group $i$ as $\mathbf{b}_{i, j}$ (see Fig. 4). Each background measurement is the average over 20 FOV acquisitions. Hence, it is considered as noise free [30]. Reasonable parameters to simulate the Gaussian noise signal $\mathbf{n}_{i, j}$ were determined from noise measurements of our MPI device.

The system matrix was recorded with drive field amplitudes of $D_{x}=18 \mathrm{mT} / \mu_{0}, D_{y}=18 \mathrm{mT} / \mu_{0}$ and $D_{z}=2 \mathrm{mT} / \mu_{0}$ at a gradient of $2 G_{x}=2 G_{y}=-G_{z}=5.5 \mathrm{Tm}^{-1} / \mu_{0}$. The two-dimensional system matrix was recorded with a delta probe of $0.8 \mu \mathrm{L}$ undiluted MM4 with an iron concentration of $2.8 \mathrm{mg}_{\mathrm{Fe}} / \mathrm{mL}$. We used a two-dimensional scanner FOV consisting of $9 \times 9$ voxels covering an area of $16 \times 16 \mathrm{~mm}^{2}$.

Two true images $\mathbf{x}_{A}^{\text {true }} \in \mathbb{R}^{9 \times 9}$ and $\mathbf{x}_{B}^{\text {true }} \in \mathbb{R}^{9 \times 9}$ were generated based on the same virtual phantom. We used two different virtual phantoms: a stenosis phantom with homogeneous 


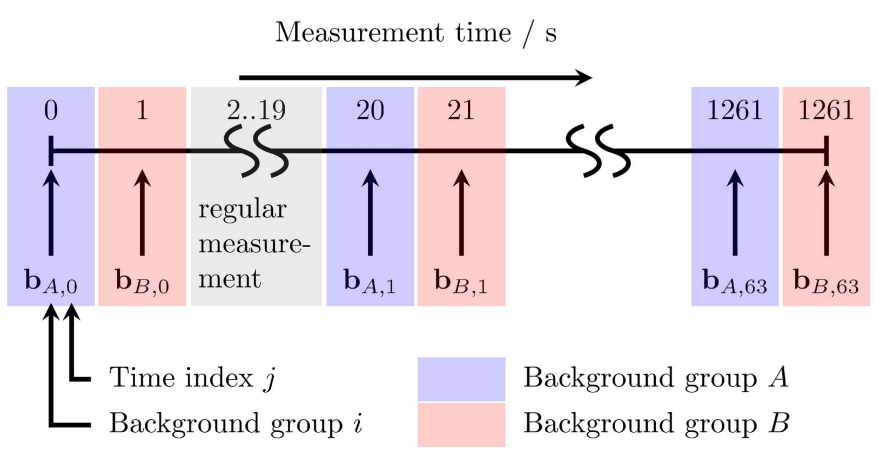

Fig. 4. Illustration of the segmentation of the background measurement used for the simulation studies. It is based on a regular measurement of about 20 min that was interrupted every $19 \mathrm{~s}$ to measure the background signal over $2 \mathrm{~s}$. Each single $2 \mathrm{~s}$ background measurement is divided in two background groups: $A$ and $B$. A single background measurement is referenced by $b_{i, j}$ with $i$ the background group and $j$ the consecutive time index.

intensity (see Fig. 5a) and a multi-gray scale phantom (see Fig. 5b). To simulate the shift of the scanner FOV, $\mathbf{x}_{A}^{\text {true }}$ and $\mathbf{x}_{B}^{\text {true }}$ were shifted in respect to each other by one row and one column, see Fig. 3. The respective true measurement signals $\mathbf{u}_{i}^{\text {true }}$ were calculated by the forward projection (2) of the virtual phantoms. According to (1), the measured consecutive background signal $\mathbf{b}_{i, j}$ as well as the synthetic noise $\mathbf{n}_{i, j}$ were added to the true image signal $\mathbf{u}_{i}^{\text {true }}$ to get the simulated measurement signal $\mathbf{u}_{i, j}$. The generated measurement signals $\mathbf{u}_{i, j}$ were reconstructed into a single concentration vector $\mathbf{c}_{j}$ with the here presented algorithm that was transformed into the image $\mathbf{x}_{j}^{\text {meas }}$ by index mapping.

1) $\beta$ Scan: To investigate the efficiency of the proposed algorithm, we examined the influence of the regularization parameter $\beta$ as well as the $\operatorname{SNR}_{\text {meas }}$ of the input data $\left(\mathbf{u}_{i, j}\right)$. The $L_{2}$ regularization is disabled, i.e. $\alpha=0$. We defined the $\mathrm{SNR}_{\text {meas }}$ as the average SNR of all frequency components ${ }^{1}$ of the complex sample signal $\mathbf{u}_{i, j}^{\text {true }}$. For each $\left(\beta, \mathrm{SNR}_{\text {meas }}\right)$ tuple, ten measurements with individually generated Gaussian noise were simulated.

To assess the image quality of the algorithm, several line profiles through the reconstructed images $\mathbf{x}_{j}^{\text {meas }}$ were examined, which are marked in Fig. 5. We used the signal to background ratio (SBR) as a measure of the background suppression. The SBR was defined for each profile as the mean signal divided by the mean background.

2) Convergence Analysis: We used the same simulation approach as for the $\beta$ scan to determine the convergence behavior of our algorithm. Here, we focused on the stenosis phantom (see Fig. 5a). Based on the results of the $\beta$ scan, we chose a fixed $\beta=0.1$ and $\alpha=0$ and varied the $\mathrm{SNR}_{\text {meas }}$ in the same way as for the $\beta$ scan.

We assess the convergence by using the mean absolute error (MAE) of the image obtained for each iteration of the L-BFGS-B minimizer on our method. The MAE measures the deviation of the reconstructed $\mathbf{c}=\left(c_{k}\right)$ from the true

\footnotetext{
${ }^{1}$ Only those frequency components that contain image information are considered by applying an SNR threshold that is based on the SNR determined from the system matrix measurement.
}

$\mathbf{c}^{\text {true }}=\left(c_{k}^{\text {true }}\right)$ concentration distribution vector and is defined as

$$
\mathrm{MAE}=\frac{1}{N_{\text {pos }}} \sum_{k=0}^{N_{\text {pos }}}\left|c_{k}^{\text {true }}-c_{k}\right|
$$

where $k$ denotes the $k$-th component of the vectors, i.e. the $k$-th voxel.

3) Comparison With Kaczmarz Algorithm: Additionally, the proposed algorithm was compared to the established Kaczmarz algorithm [25] in a simulation study. To this end, for each time step $j$ of the background measurement $\mathbf{b}_{i, j}$, we reconstructed the concentration distribution $\mathbf{c}_{j}$ with the Kaczmarz algorithm as well as the here proposed one. For the Kaczmarz algorithm, the average of the measurement vectors $\mathbf{u}_{A, j}^{\mathrm{K}}$ and $\mathbf{u}_{B, j}^{\mathrm{K}}$ without a shift of the scanner FOV was used. For the joint reconstruction algorithm, the scanner FOV was shifted between $\mathbf{u}_{A, j}^{\mathrm{L}}$ and $\mathbf{u}_{B, j}^{\mathrm{L}}$. The background signal as well as the noise was added as described before. Hence, the simulated measurement vectors for background measurement group $i$ at time step $j$ are given by:

$$
\begin{aligned}
\mathbf{u}_{i, j}^{\mathrm{K}} & =\mathbf{S c}(\mathbf{r})+\mathbf{b}_{i, j}+\mathbf{n}_{i, j} \\
\mathbf{u}_{A, j}^{\mathrm{L}} & =\mathbf{S c}\left(\mathbf{r}_{A}\right)+\mathbf{b}_{A, j}+\mathbf{n}_{A, j} \\
\mathbf{u}_{B, j}^{\mathrm{L}} & =\mathbf{S c}\left(\mathbf{r}_{B}\right)+\mathbf{b}_{B, j}+\mathbf{n}_{B, j}
\end{aligned}
$$

For the Kaczmarz reconstruction, we tested two common cases of conventional background subtraction during simulation:

1) Subtraction of a background that is measured directly before the measurement series.

2) Subtraction of a background that is linearly interpolated between two background measurements, which were recorded directly before and after the measurement series.

The iterative Kaczmarz reconstruction was executed with 100 iterations and no $L_{2}$ regularization to reduce the space of reconstruction parameters.

For the joint reconstruction method, we evaluated two similar cases for seeding of the algorithm's parameter $\mathbf{b}_{\text {init }}$ :

1) Seeding with the previously fitted background. For the first image, we use a background recorded directly before the sample measurement.

2) Seeding with the linearly interpolated background between two background measurements, which were recorded directly before and after the measurement series.

We compared both approaches by calculating the SBR of the chosen line profiles (see Fig. 5a and Fig. 5b) of the reconstructed images. All input measurements have an $\mathrm{SNR}_{\text {meas }}$ of 23. For comparability with the Kaczmarz results, the $L_{2}$ regularization is disabled as well. For both seeding methods, we used the same $\beta=0.1$.

4) Dependency on the Scanner FOV Shift: Furthermore, the dependency of the performance of the joint reconstruction algorithm on the chosen shift of the scanner FOV was investigated. For this purpose, the time series simulation of the stenosis phantom was also executed without a shift as well as with a one voxel shift in $x$ or $y$ direction, respectively. 


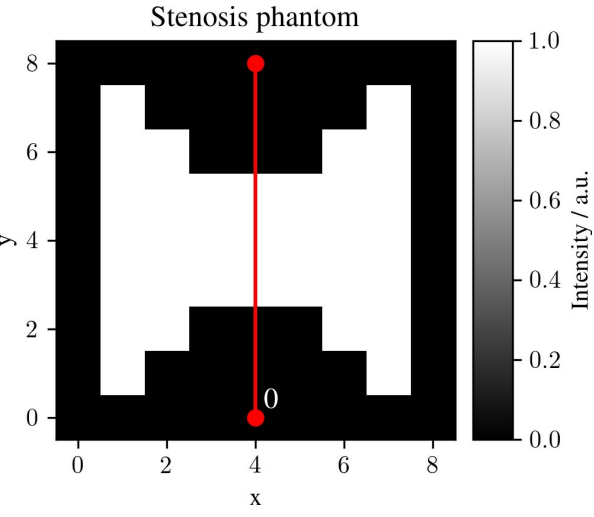

(a)

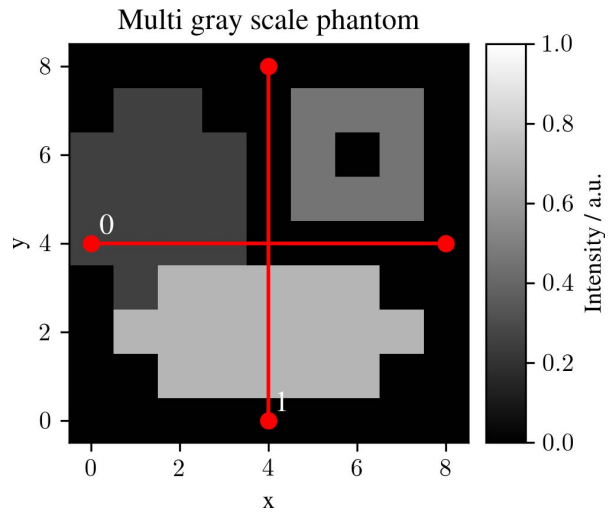

(b)

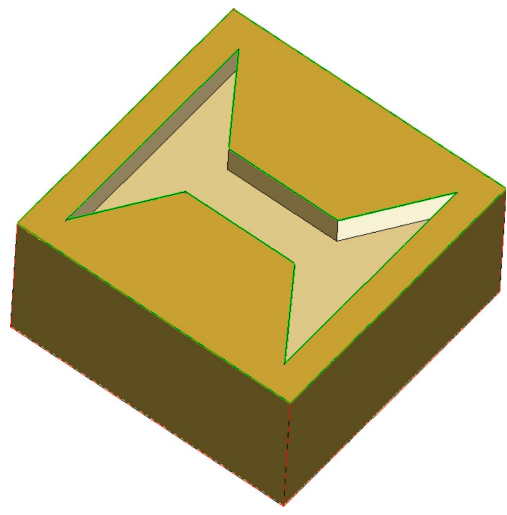

(c)

Fig. 5. Stenosis (a) and multi-gray value (b) phantoms for the simulations studies. Both images have the same gray scale. The red lines mark the voxels that are used to calculate the signal to background ratio (SBR). (c) Shows the 3D drawing of the stenosis phantom used for the measurement study.

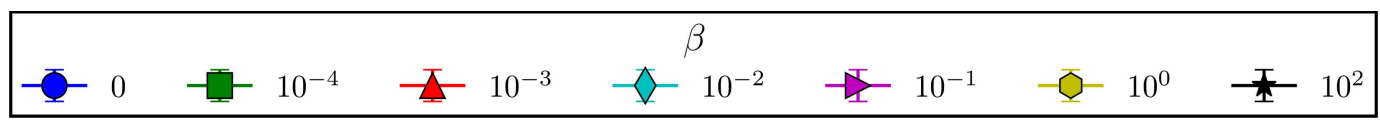

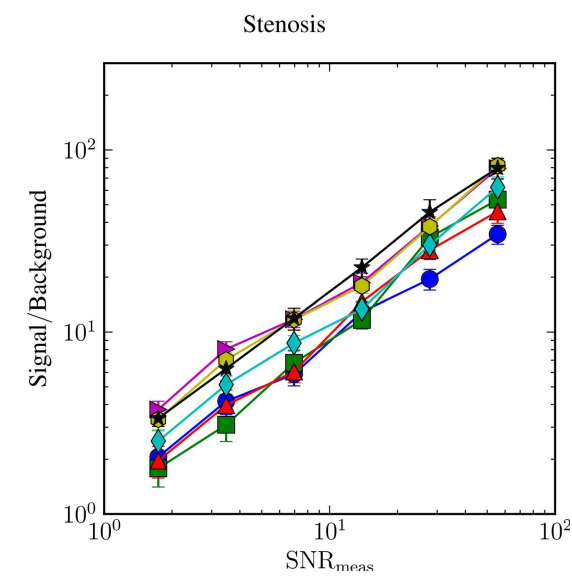

(a)

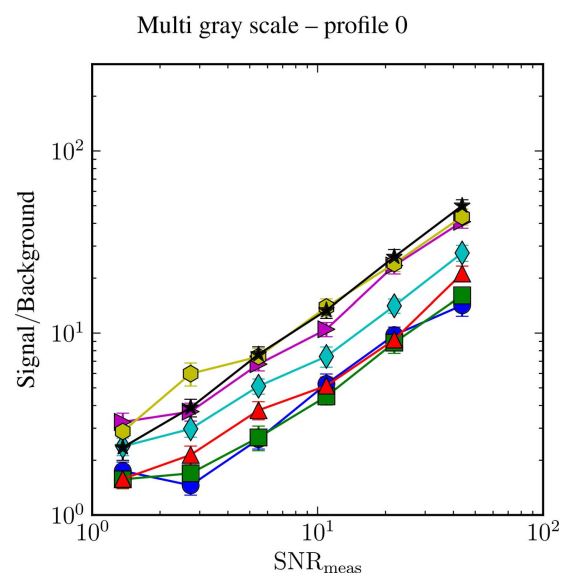

(b)

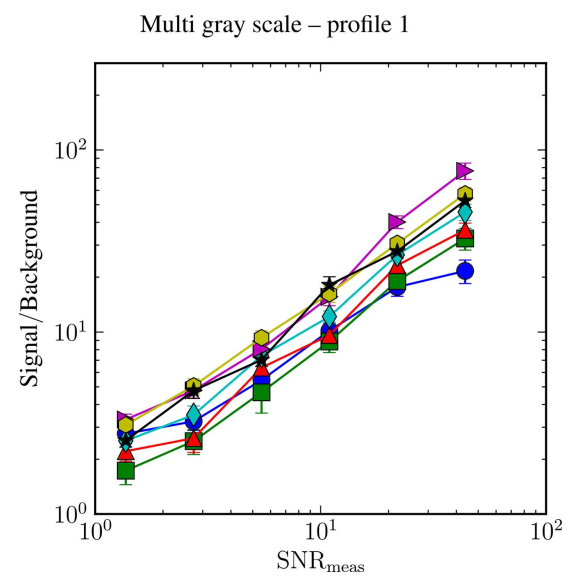

(c)

Fig. 6. Extracted SBR from simulated image measurements for the joint reconstruction algorithm as a function of the measurement SNR (SNRmeas) and $\beta$. (a) Stenosis phantom. (b) Multi-gray scale phantom line profile 0 (low intensity area). (c) Multi-gray scale phantom line profile 1 (high intensity area).

\section{E. Measurement}

The potential of the joint reconstruction method was studied on an actual stenosis phantom measurement (cf. Fig. 5c). The phantom was filled with $32 \mu \mathrm{L}$ of diluted MM4 at a concentration of $44 \mu \mathrm{g}_{\mathrm{Fe}} / \mathrm{mL}$.

We used the same drive field and gradient settings as for the acquisition of the system matrix in the simulation studies. However, here we used a system matrix with a higher resolution of $25 \times 25$ voxels covering the same area of $16 \times 16 \mathrm{~mm}^{2}$ as before, because it is closer to the typically used resolutions [2], [14], [31] and fewer images need to be reconstructed than for the simulation studies. We shifted the scanner FOV by 1 voxel in $x$ direction. The shift is executed by moving the phantom with the same robot that is normally used to measure the system matrix.

After a warm up period of about 5 minutes, an initial background was measured. After additional 2 minutes, the actual phantom measurement was recorded and was followed by a final background measurement 11 min later. For joint reconstruction, the initial background parameter $\mathbf{b}_{\text {init }}$ was initialized with method 2 and $\beta=0.1$ was used. For the Kaczmarz reconstruction, the background was subtracted with method 2 and the measurement was reconstructed with 10 iterations. For both methods, the same $L_{2}$ regularization value $\alpha=0.1$ was used.

\section{Results}

\section{A. Simulation Studies}

The reconstruction of a $9 \times 9$ voxel image with the joint reconstruction algorithm took about $10 \mathrm{~s}$ on an Intel Core i7-6700 quad-core at $3.4 \mathrm{GHz}$. In comparison, the Kaczmarz reconstruction took about $100 \mathrm{~ms}$ (100 iterations) on the same computer.

1) $\beta$ Scan: Fig. 6 shows the SBR in dependence on $\mathrm{SNR}_{\text {meas }}$ and the reconstruction parameter $\beta$ of (5). Due to the limited SNR of the system matrix measurement, the simulation is 


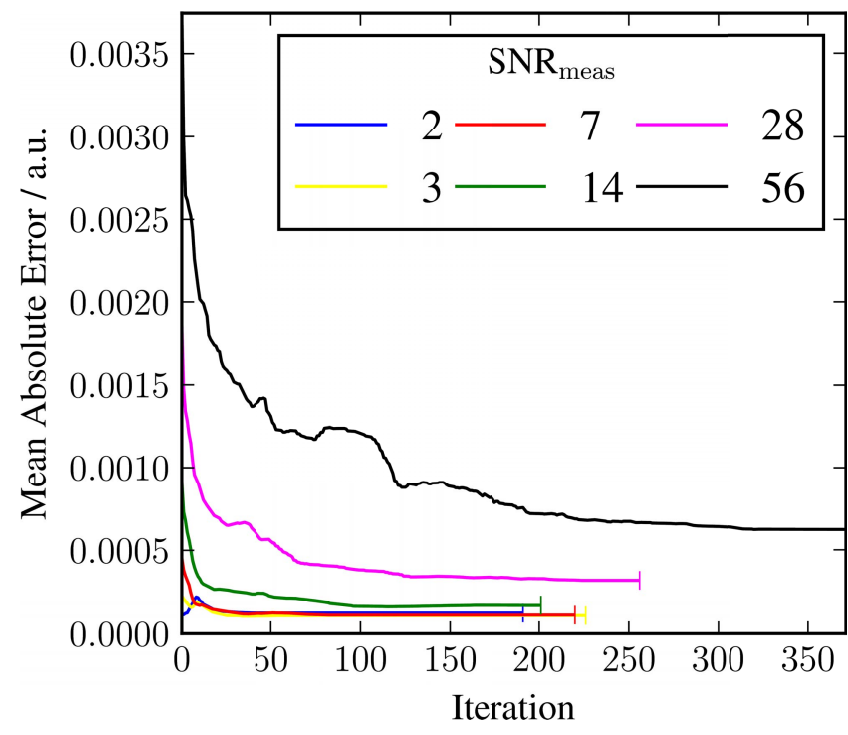

Fig. 7. Mean Absolute Error (MAE) between the estimated and the true tracer distribution in dependence on the number of iterations of the joint tracer and background reconstruction from simulation. The evolution of the MAE is shown for SNR meas values: 2, 3, 7, 14, 28, and 56. For higher values of SNRmeas, the value of each voxel filled with tracer is higher, too. This leads to a higher MAE for simulations with higher SNRmeas.

limited to an $\mathrm{SNR}_{\text {meas }}$ of about 60 for the Stenosis phantom. For higher values of $\mathrm{SNR}_{\text {meas }}$, the influence of the system matrix noise on the reconstructed images increases. For all chosen profile lines, a lower value of $\beta$ corresponds to a lower SBR. The results for values of $\beta$ from 0.1 to 100 look similar. For $\beta=0.1$, a good SBR is achieved for a wide range of values of $\mathrm{SNR}_{\text {meas }}$.

2) Convergence Analysis: Fig. 7 shows the MAE in dependence on the iteration and the $\mathrm{SNR}_{\text {meas }}$. The MAE is higher for higher values of $\mathrm{SNR}_{\text {meas }}$ because the absolute intensity of voxels filled with tracer is higher. For all used values of $\mathrm{SNR}_{\text {meas }}$, a convergence of the MAE is visible. The MAE is not a monotone function of the number of iterations. Towards lower values of $\mathrm{SNR}_{\text {meas }}$, the number of required iterations lowers from 370 , i.e. $\mathrm{SNR}_{\text {meas }}=56$, to about 200 , $\mathrm{SNR}_{\text {meas }}<28$.

3) Comparison With Kaczmarz Algorithm: Fig. 8d, Fig. 8e and Fig. 8f show the reconstruction results as well as the line profile 0 (see Fig. 5b) of the time series simulation for time step 14 of the Kaczmarz algorithm as well as the joint reconstruction method for the background seeding/correction method two. The structures in the image being reconstructed with the joint reconstruction approach (see Fig. 8e) are well distinguishable, whereas the images reconstructed with the Kaczmarz algorithm combined with the established subtraction scheme (see Fig. 8d) are very noisy and exhibit a lower contrast. This observation is also supported by the matching line profiles (see Fig. 8f). The difference in the resulting image quality is also reflected in the achieved SBR of 38.4 for the joint reconstruction method and 11.8 for the Kaczmarz method.

The resulting evolution of the SBR over time for all line profiles of the test images (see Fig. 5a and Fig. 5b) is shown in Fig. 8a, Fig. 8b and Fig. 8c. To ease the comparison between
TABLE I

AVERAGE RATIO $r_{\text {SBR }}=$ SBR $_{\text {JOINT RECONSTRUCTION }} /$ SBR $_{\text {KACZMARZ }}$ OF THE IMAGE SBR IN THE TIME SERIES RECONSTRUCTION FOR THE DEFINED LINE PROFILES. A SHIFT OF THE SCANNER FOV IN EACH DiRECTION By ONE Voxel IS USED. TO MAKE THE average more Robust, All sbr Values Above 1000 WERE DISCARDED FOR ITS CALCULATION

\begin{tabular}{lllr}
\hline Image & $\begin{array}{l}\text { Line } \\
\text { profile }\end{array}$ & $\begin{array}{l}\text { Background } \\
\text { seeding/correction }\end{array}$ & $r_{\mathrm{SBR}}$ \\
\hline Multi-gray scale & 0 & 1 & $3.2 \pm 0.6$ \\
& & 2 & $6 \pm 2$ \\
& 1 & 1 & $5 \pm 1$ \\
Stenosis & & 2 & $10 \pm 1$ \\
& 0 & 1 & $4.2 \pm 0.8$ \\
& & 2 & $16 \pm 3$ \\
\hline
\end{tabular}

both reconstruction methods, the ratio of the average SBR is given in Table I. For the averaging of the SBR, the line profiles with an extraordinary high SBR above 1000 are discarded to make the calculation of the mean as well as its standard deviation more robust.

4) Dependency on the Scanner FOV Shift: Fig. 9 shows the average SBR for each line profile and reconstruction method. For the stenosis phantom, the dependency on the applied shift of the scanner FOV is shown, too. For the simulation of the Kaczmarz algorithm, no shift of the scanner FOV is applied. Therefore, the SBR values determined from the Kaczmarz reconstruction are only shown for the case of a shift in $x$ and $y$ direction. Besides the case of no shift of the scanner FOV combined with seeding method 1, the SBR gained from reconstruction with our algorithm is higher than for the Kaczmarz reconstructions.

\section{B. Measurement}

The reconstruction of a $25 \times 25$ voxel image with our algorithm took about $40 \mathrm{~s}$, while the reconstruction with the Kaczmarz algorithm took about $0.1 \mathrm{~s}$ (10 iterations). Fig. 10a shows the measured stenosis phantom data reconstructed with the Kaczmarz algorithm. For the joint reconstruction method, the resulting reconstructed image is shown in Fig. 10b. The border of the actual phantom is marked with a blue line. In both images, the profiles investigated for the SBR calculation are marked with red (background region) and green (signal region) lines. Furthermore, the line profiles are numbered consistently from 0 to 3 and the related line profiles are shown in Fig. 10c, Fig. 10d, Fig. 10e and Fig. 10f. Both reconstructed images are scaled individually to use the full range from 0.0 to 1.0 . The SBR values for the single line profiles are given in Table II.

\section{Discussion}

In this work, we presented a method that allows to reduce the quasi-static image domain background of MPI during reconstruction. Thereto, the tracer distribution as well as the background signal are jointly estimated by our reconstruction algorithm. This is achieved by modifying the image acquisition scheme to execute a small shift of the scanner FOV between 


\begin{tabular}{|llll|}
- & Joint reconstruction (Type 1) & - & Kaczmarz (Type 1) \\
- & Joint reconstruction (Type 2) & - & Kaczmarz (Type 2) \\
\hline
\end{tabular}

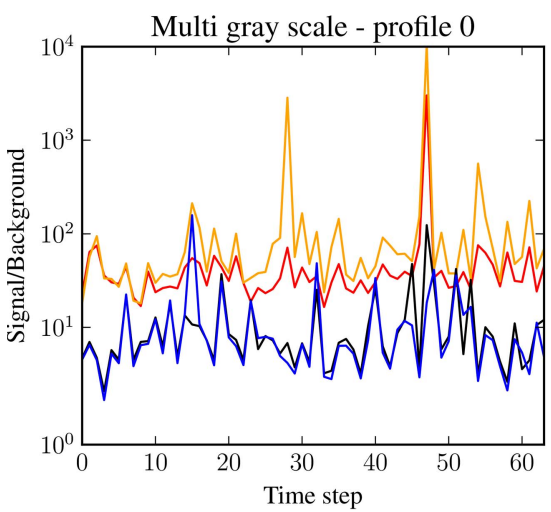

(a)

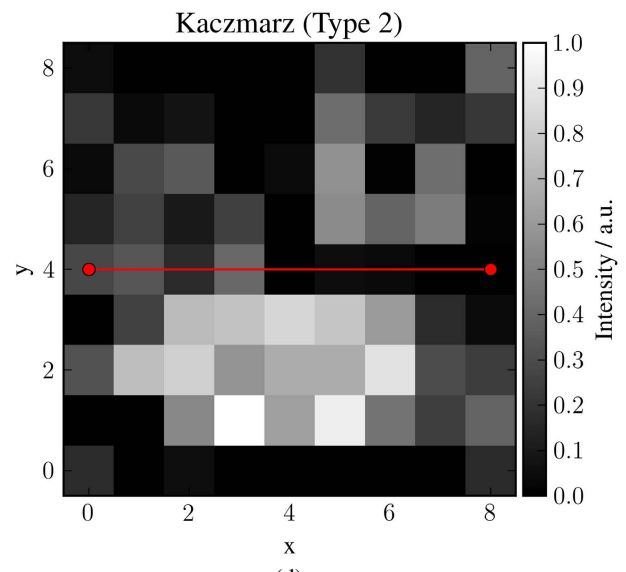

(d)

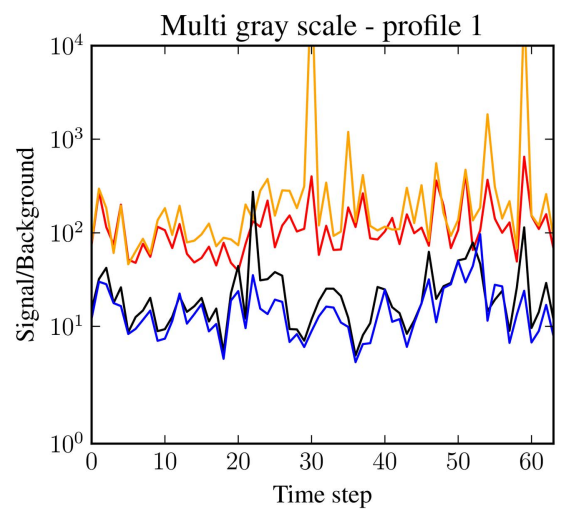

(b)

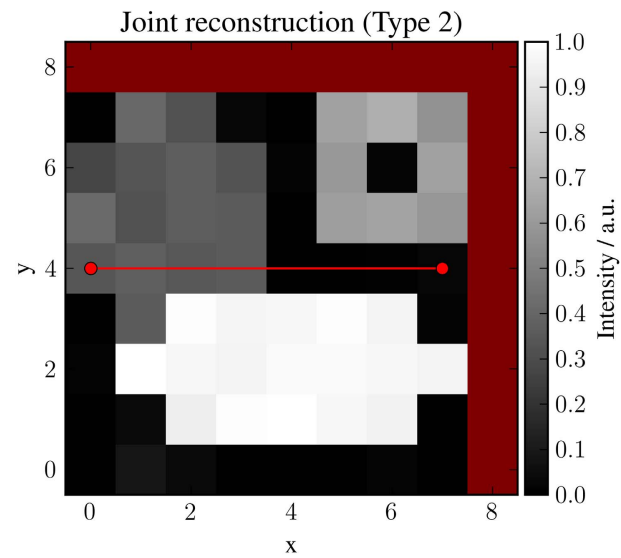

(e)

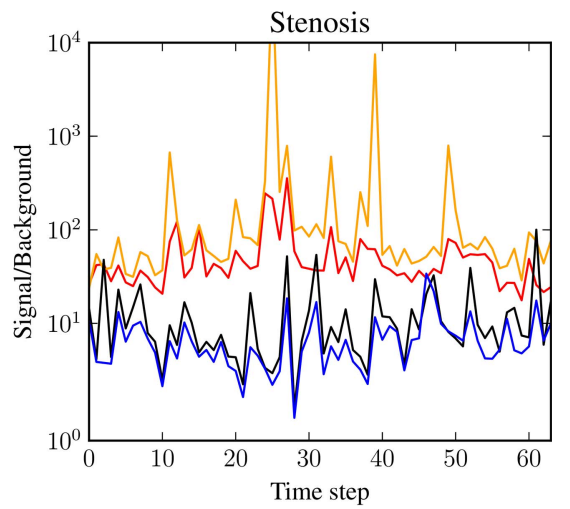

(c)
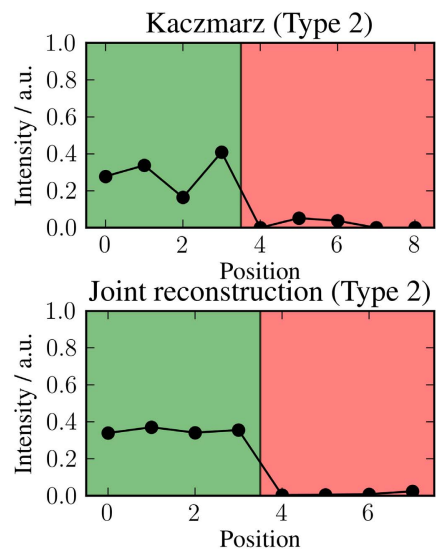

(f)

Fig. 8. (a-c) The SBR as a function of the simulated time step from the time series simulation is shown for both reconstruction techniques for the multi-gray scale phantom as well as the simple stenosis phantom. (a) Line profile 0 of the multi-gray scale phantom. (b) Line profile 1 of the multi-gray scale phantom. (c) Line profile 0 of the stenosis phantom. (d-e) Reconstructed images of time step 14 from the simulated time series reconstruction of the multi-gray scale phantom for background subtraction/seeding technique 2 . The images are individually scaled to use the full dynamic. (d) Kaczmarz reconstruction of the simulated signal. (e) Joint reconstruction of the simulated signal. The area lost due to the shift of the scanner FOV is marked in red. (f) Line profiles 0 of the reconstructed images. The SBR for the Kaczmarz and joint reconstruction is 11.8 and 38.4 , respectively.

TABLE II

SBR of the LiNe PROFILES OF THE IMAGES RECONSTRUCTED From the MEASUREMENT OF THE StENOSIS PHANTOM FILLED WITH DILUTED MM4 $\left(44 \mu \mathrm{g}_{\mathrm{FE}} / \mathrm{mL}\right)$

\begin{tabular}{lll}
\hline $\begin{array}{l}\text { Line } \\
\text { profile }\end{array}$ & Reconstruction method & SBR \\
\hline 0 & Kaczmarz & 17.6 \\
& Joint Reconstruction & 25.0 \\
1 & Kaczmarz & 11.3 \\
& Joint Reconstruction & 44.4 \\
2 & Kaczmarz & 10.5 \\
& Joint Reconstruction & 17.3 \\
3 & Kaczmarz & 19.3 \\
& Joint Reconstruction & 28.9 \\
\hline
\end{tabular}

consecutive volume measurements. We did a first characterization of the algorithm. In simulations, we investigated the convergence of the algorithm as well as the dependence of the SBR on the new reconstruction parameter $\beta$ (see (5)) and the shift of the scanner FOV. Also, we compared our algorithm with the established Kaczmarz algorithm. Besides simulations, we also compared our method to the Kaczmarz algorithm with an actual static MPI phantom measurement.

From the simulations, we found that the regularization factor for the euclidean difference between the initial $\mathbf{b}_{\text {init }}$ and the fitted $\mathbf{b}$ background just has a minor implication on the resulting SBR. It was shown that the SBR increases for higher values of $\beta$. However, an increased $\beta$ has the disadvantage that the penalty for large changes in the background increases. This would render the approach less useful for extracting a strongly changing background. On the contrary, a too loose value of $\beta$ might lead to a strong susceptibility to noise. A mismatch between background and particle contribution might be the consequence.

The convergence analysis showed that for all considered values of $\mathrm{SNR}_{\text {meas }}$ the difference, i.e. MAE, between the true and the reconstructed image reduces with increasing number of iterations. However, during the first few ten iterations the MAE might increase again for few iterations. Hence, it seems reasonable to use the maximum norm of the gradient, which is used by the L-BFGS-B algorithm, as a stopping criterion. 


\begin{tabular}{|cccc|}
\hline I & Joint reconstruction - 1 & $\mathbf{I}$ & Kaczmarz - 1 \\
里 & Joint reconstruction - 2 & 王 & Kaczmarz - 2 \\
\hline
\end{tabular}

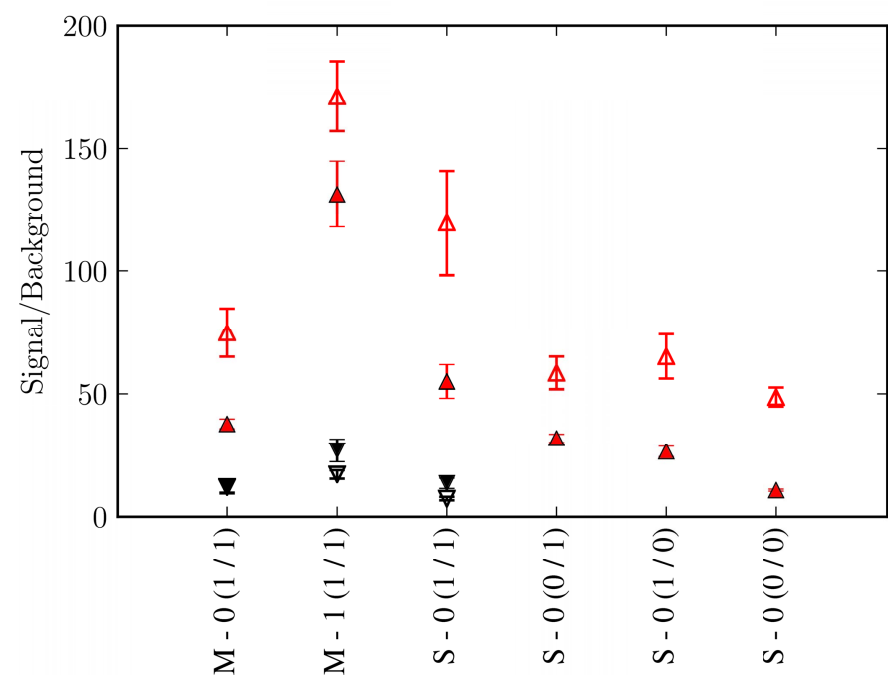

Fig. 9. The average SBR of the chosen profile lines for all reconstruction methods on the time series simulation. An open or filled marker means that for the background correction respectively seeding a linearly interpolated respectively primarily measured background is used. The horizontal axis is labeled with the phantom name, i.e. M for the multi-gray scale and $\mathrm{S}$ for the stenosis phantom, followed by the profile line number and the shift used for the joint reconstruction method, which is encoded as $(x / y)$ direction. For the stenosis phantom, the Kaczmarz results are the same for all shifts. So these are only shown for "S - $0(1 / 1)$ ".

The maximum of the gradient norm is a measure for how large the next change of the variables is. So if the changes get smaller and smaller, the solution seems to converge. However, it needs to be investigated how the best choice of this parameter depends on the data to reconstruct. For example, the best choice of the number of iterations, i.e. the stopping criterion, used for the Kaczmarz algorithm depends on the object under examination. So, the complexity of using these algorithms does not differ in regards of the stopping criterion selection.

For the comparison of this method to the established Kaczmarz reconstruction algorithm, we used simulated as well as measured MPI data. Both (see Fig. 8 and Fig. 10) showed that our method improves the resulting image quality. Since both reconstruction methods need an initial guess of the actual background of the sample measurement to return good results, we examined two techniques to approximate the initial guess: 1 . a single background measurement before the sample measurement and 2. a measurement before as well as after the sample measurement with linear interpolation in between. For method 1, we found in simulations that a gain of about 4 (see Table I) in terms of the SBR is recognizable for the joint reconstruction approach for all investigated line profiles of the two simulation phantoms (see Fig. 5). An even higher gain of about 10 is achieved if method 2 is used for background seeding (see Table I). Table I also shows that the achievable SBR gain is reduced for line profiles of the reconstructed image with a reduced signal intensity, i.e. line profile 0 of the multi-gray scale phantom.
Besides the background seeding of the proposed algorithm, also the used spatial shift of the scanner FOV between the recorded volumes has an impact on the SBR improvement (see Fig. 9). Even without a shift of the scanner FOV between the consecutive measurements, see column " $S-0(0 / 0)$ " in Fig. 9, a seven times higher SBR gain was observed for the joint reconstruction than for the Kaczmarz algorithm. Without a shift between consecutive volumes, we assumed that the algorithm converges to an average of both contributing measurements. Hence, just the noise level would be lowered by $\sqrt{2}$. This assumption is based on the consideration that without a shift, the algorithm cannot distinguish between the non-altered particle signal and the static background. On the contrary, with increasing prior knowledge, i.e. by shifting the scanner FOV in one or more directions, the separation of background and particle signal should enhance. This is due to the fact that with the increasing number of spatial directions considered by the algorithm, also the influence on the signal in the three orthogonal receive channels increases. In turn, this results in larger differences between the acquired signals which eases the separation of background and particle signal. Hence, the simulation results show that with an increasing number of axes, which are considered for the shift of the FOV, the SBR gain increases with our method. Thus, the highest SBR gain, i.e. 16 for the stenosis phantom, was achieved by a shift in both directions, see column "S - 0 (1/1)" in Fig. 9. Hence, this gain probably results from the successful background estimation.

For the measured phantom filled with diluted MM4, i.e. $44 \mu \mathrm{g} F / \mathrm{mL}$, the structure is best reproduced by our method (see Fig. 10). This is also reflected by the values of the SBR along the selected line profiles, which is on average a factor of $2.0 \pm 0.5$ higher for the joint reconstruction method (see Table II). However, this is a factor of $5 \pm 2$ worse than the SBR gain determined from the reconstruction of the time series simulations (see Table I).

This difference in the determined SBR gain between simulation and measurement might be caused by a misalignment of the phantom and the system matrix grid. This alignment is challenging due to the small grid size of $640 \mu \mathrm{m}$ but was executed as well as technically possible in the used setup. Furthermore, the used simulation technique neglects position dependent background, which might arise from very small contaminations with magnetic material from the manufacturing process of the phantom as well as the sample holder.

Another source of the difference between simulation and measurement might be the system matrix measurement. For the system matrix measurement, the regular background subtraction with linear interpolation of the background signal was used. Although the background measurement was executed every $20 \mathrm{~s}$, this might be insufficient for a linear approximation of the background change. Hence, the system matrix might be incorrect for at least some positions. This did not pose a problem for the simulations, because the same system matrix was used for signal generation as well as image reconstruction. While avoiding the above mentioned problem in simulation, the previously measured system matrix does not necessarily fit perfectly to the measurement. 


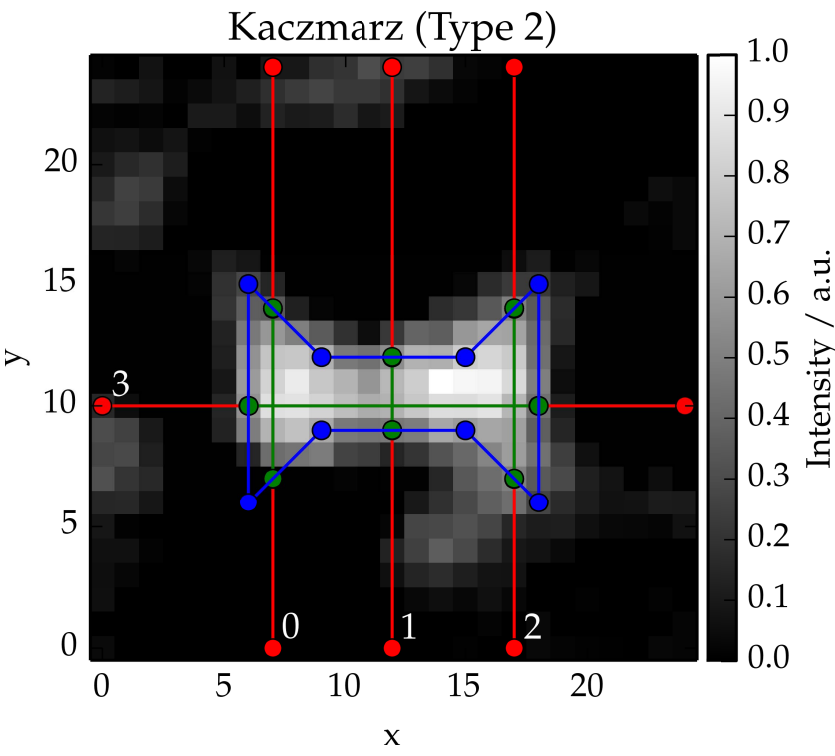

(a)

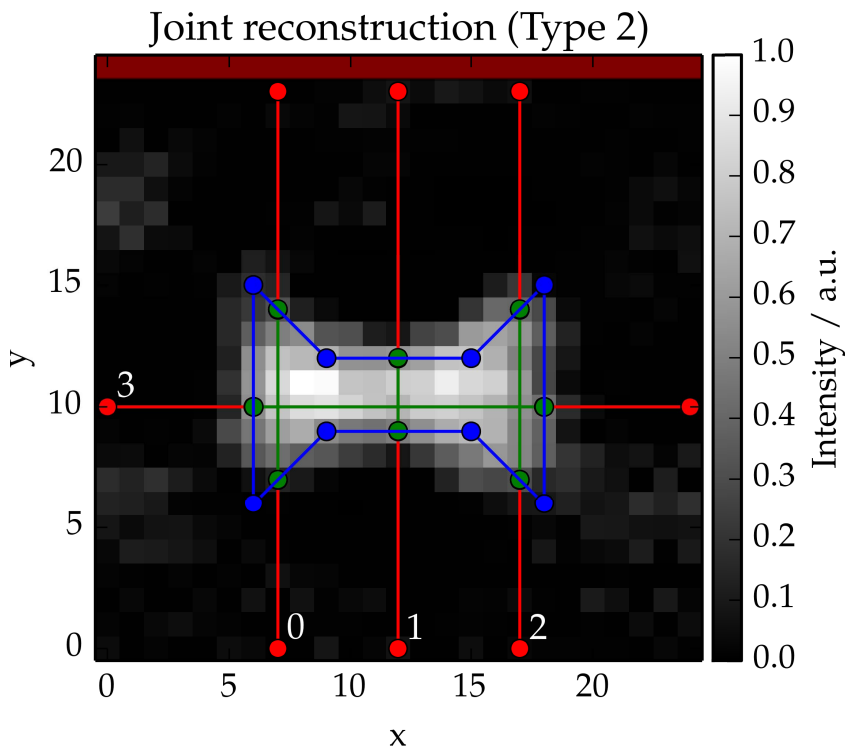

(b)

\section{$\bullet$ Kaczmarz (Type 2) $\bullet$ Joint reconstruction (Type 2)}

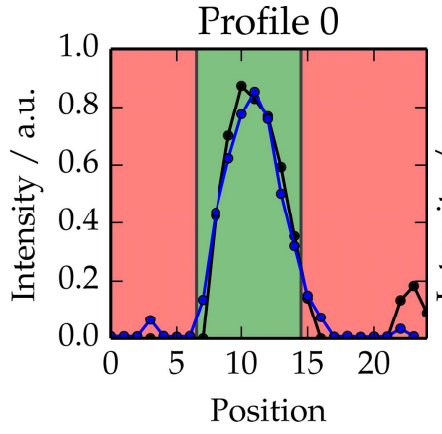

(c)

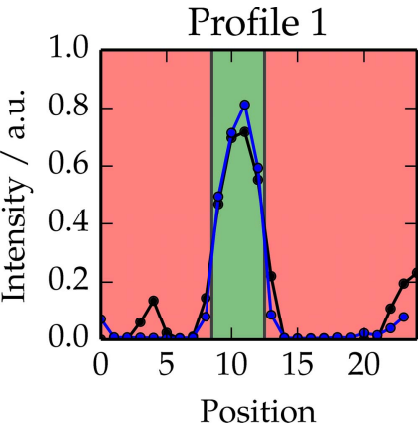

(d)

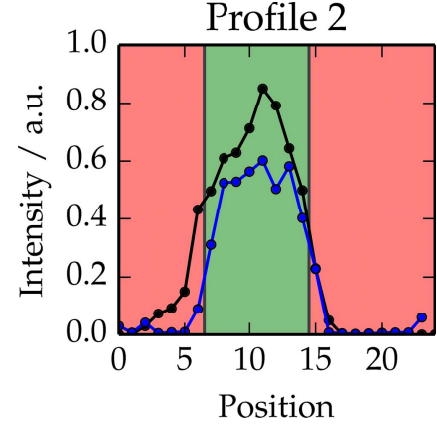

(e)

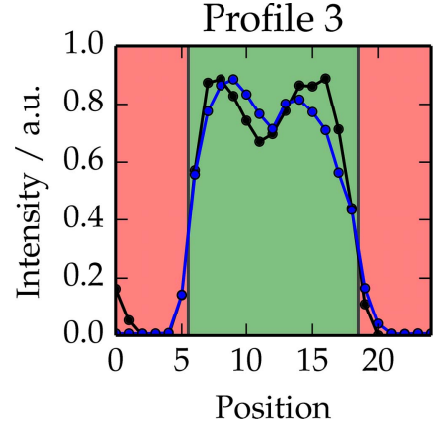

(f)

Fig. 10. Images reconstructed from the measurement of a stenosis phantom filled with diluted MM4 $\left(44 \mu \mathrm{F}_{\mathrm{Fe}} / \mathrm{mL}\right)$. The images are individually scaled to be represented by gray scale values between 0 and 1. (a) Reconstructed image for the conventional Kaczmarz reconstruction with background subtraction technique (2). (b) Reconstruction applying the presented joint reconstruction algorithm and seeding technique (2). (a-b) The true shape and position of the phantom is marked with blue lines. Line profiles are marked and numbered in images with red lines, green sections along the line profiles mark the chosen signal region. (c-f) From both reconstructed images line profiles are presented as described in the legend. The signal regions are marked in green and the background regions in red.

A potential drawback of our method that we did not yet address might be its susceptibility to large changes of the observed tracer distribution between two consecutively recorded volumes. Because in this case it is not obvious if the presented algorithm correctly assigns the tracer signal to the image and not the background. Thereto, the stability of the algorithm regarding strong changes needs to be further investigated before it can be used in dynamic studies where this might be an issue. However, for small changes of the tracer distribution, we assume that a smearing comparable to that of a reduced volume acquisition rate occurs. Hence, the frame rate is reduced by a factor of two because two volumes are fused to a single background corrected one. In commercially available MPI devices [31], this are still effective 23.21 volumes per second if focus fields can be used for the FOV shift. Nevertheless, the reduced volume rate should be still sufficient for imaging the beating heart of an anesthetized mouse, which is between 160 and 460 beats per minute [32]. Hence, the achievable frame rate is still reasonable for several dynamic studies if the tracer distribution changes slowly enough. Besides theoretical considerations, it still needs to be shown that this method is actually capable to reduce the background in dynamic studies.

Another challenge for this algorithm is that the time required to reconstruct one frame is increased by a factor of about 400 in comparison to the established Kaczmarz reconstruction, i.e. $40 \mathrm{~s}$ compared to $0.1 \mathrm{~s}$. For a typical in-vivo measurement of $1 \mathrm{~min}$, i.e. 2760 volumes, this results in a total reconstruction 
time of approximately $31 \mathrm{~h}$ in contrast to $5 \mathrm{~min}$ for the conventional reconstruction. However, the algorithm is currently implemented in Python and no optimization has been applied. So we see the potential to reduce the required computational afford by using $\mathrm{C}++$ with an optimized library that makes use of modern multi-core CPUs or even GPUs. It is also reasonable to look in different minimizers which might fit better to the here presented reconstruction rule than the currently used L-BFGS-B algorithm.

By applying our method only to selected volumes, the impact on the total reconstruction time as well as the temporal resolution can be reduced. For example, during a prolonged measurement our method could be applied only every 20th second $^{2}$ to estimate the true background. In between, the measurements are corrected with the previously estimated background and are reconstructed with the conventional Kaczmarz algorithm. For a measurement of 1 min with 46 volumes per second, this results in a reconstruction time of about 6 min which is a prolongation of about $20 \%$ compared to a mere Kaczmarz reconstruction.

There exist also other approaches to cope with the dynamic background in MPI. In [11], the MPI background was split in a static and a dynamic part. The frequency components contributing to the dynamic background are removed for reconstruction. However, these frequencies also carry imaging information which our method can still use for reconstruction. Von Gladiss et al. [18] proposed another method to jointly estimate the background and the particle concentration that is called background extended system matrix reconstruction (BESM). This is achieved by extending the system matrix by one additional background "pixel". Hence, it is nearly as fast as the established reconstruction technique. To cope with a changing background a scaling parameter is introduced. Hence, it is bound to the spectral structure of the previously measured background. The here presented method, however, uses the equality between well known shifted tracer distributions to determine the background and uses the previously measured background only for seeding. Besides, the parameter $\beta$ allows to influence how strong the initial background shall resemble the fitted one. Thus, our method can cope with more complex background changes, i.e. a change of the spectral structure.

If we can successfully evaluate our algorithm for dynamic processes and reduce the required computation time, it will be useful for all prolonged MPI measurements. For example, interventional imaging [7] requires a prolonged MPI measurement. In this case, it is unfeasible to interrupt the actual measurement, remove the patient from the device and then remeasure the background signal. However, for best visual guidance during intervention, the imaging quality is crucial, too. Both points are addressed by our method.

\section{CONCLUSION}

In this paper, we proposed and investigated a new image reconstruction method using a shifted acquisition scheme to

\footnotetext{
${ }^{2}$ System matrix measurements are usually interrupted for background measurement in this interval.
}

reconstruct the tracer distribution as well as the background simultaneously. The method uses the background as a fit parameter. Two-dimensional simulations as well as measurements have been studied in this paper and show a distinct increase of the signal to background ratio (SBR). The reported SBR gain of 2 for actual measurements and 10 for simulations is clearly visible in the reconstructed images. The gain in SBR evokes from a reduction of the available frame rate (by a factor of 2), a slight reduction of the available FOV (by one voxel), as well as the increased computation time for the reconstruction. However, for the here used MPI device as well as the one marketed by Bruker, the frame rate reduction results in 23.31 volumes per second. This should be still sufficient to image the beating heart of an anesthetized mouse.

\section{ACKNOWLEDGMENT}

The authors would like to thank Philips for transferring their first MPI scanner to us. Furthermore, The authors want to thank Bernhard Gleich and Jürgen Rahmer for the fruitful discussions as well as Nicolas Groß-Weege and Teresa Nolte for language editing and proofreading.

\section{REFERENCES}

[1] B. Gleich and J. Weizenecker, "Tomographic imaging using the nonlinear response of magnetic particles," Nature, vol. 435, pp. 1214-1217, Jun. 2005. [Online]. Available: http://doi.org/10.1038/nature03808

[2] J. Weizenecker, B. Gleich, J. Rahmer, H. Dahnke, and J. Borgert, "Three-dimensional real-time in vivo magnetic particle imaging," Phys. Med. Biol., vol. 54, no. 5, pp. L1-L10, Mar. 2009. [Online]. Available: http://doi.org/10.1088/0031-9155/54/5/L01

[3] B. Zheng et al., "Magnetic particle imaging tracks the long-term fate of in vivo neural cell implants with high image contrast," Sci. Rep., vol. 5, Sep. 2015, Art. no. 14055. [Online]. Available: http://doi.org/10.1038/srep14055

[4] B. Zheng et al., "Quantitative magnetic particle imaging monitors the transplantation, biodistribution, and clearance of stem cells in vivo," Theranostics, vol. 6, no. 3, pp. 291-301, Jan. 2016. [Online]. Available: http://doi.org/10.7150/thno.13728

[5] X. Y. Zhou et al., "First in vivo magnetic particle imaging of lung perfusion in rats," Phys. Med. Biol., vol. 62, no. 9, pp. 1-12, Feb. 2017. [Online]. Available: http://doi.org/10.1088/1361-6560/aa616c

[6] E. Y. Yu et al., "Magnetic particle imaging: A novel in vivo imaging platform for cancer detection," Nano Lett., vol. 17, no. 3, pp. 1648-1654, Mar. 2017. [Online]. Available: http://doi.org/10. 1021/acs.nanolett.6b04865

[7] J. Rahmer, D. Wirtz, C. Bontus, J. Borgert, and B. Gleich, "Interactive magnetic catheter steering with 3-D real-time feedback using multi-color magnetic particle imaging," IEEE Trans. Med. Imag., vol. 36, no. 7, pp. 1449-1456, Jul. 2017. [Online]. Available: http://doi.org/10.1109/TMI.2017.2679099

[8] J. Weizenecker, B. Gleich, and J. Borgert, "Magnetic particle imaging using a field free line," J. Phys. D, Appl. Phys., vol. 41, no. 10, p. 105009, May 2008. [Online]. Available: http://doi.org/10.1088/0022$3727 / 41 / 10 / 105009$

[9] K. Bente, M. Weber, M. Graeser, T. F. Sattel, M. Erbe, and T. M. Buzug, "Electronic field free line rotation and relaxation deconvolution in magnetic particle imaging," IEEE Trans. Med. Imag., vol. 34, no. 2, pp. 644-651, Feb. 2015. [Online]. Available: http://doi.org/10.1109/TMI.2014.2364891

[10] J. J. Konkle, P. W. Goodwill, O. M. Carrasco-Zevallos, and S. M. Conolly, "Projection reconstruction magnetic particle imaging," IEEE Trans. Med. Imag., vol. 32, no. 2, pp. 338-347, Feb. 2013. [Online]. Available: http://doi.org/10.1109/TMI.2012.2227121

[11] K. Them et al., "Sensitivity enhancement in magnetic particle imaging by background subtraction," IEEE Trans. Med. Imag., vol. 35, no. 3, pp. 893-900, Mar. 2016. [Online]. Available: http://doi.org/10.1109/TMI.2015.2501462 
[12] T. Knopp, K. Them, M. Kaul, and N. Gdaniec, "Joint reconstruction of non-overlapping magnetic particle imaging focusfield data," Phys. Med. Biol., vol. 60, no. 8, pp. L15-L21, Mar. 2015. [Online]. Available: http://doi.org/10.1088/0031-9155/ 60/8/L 15

[13] J. Rahmer, A. Halkola, B. Gleich, I. Schmale, and J. Borgert, "First experimental evidence of the feasibility of multi-color magnetic particle imaging," Phys. Med. Biol., vol. 60, no. 5, pp. 1775-1791, Feb. 2015. [Online]. Available: http://doi.org/10.1088/0031-9155/60/ $5 / 1775$

[14] J. Franke et al., "System characterization of a highly integrated preclinical hybrid MPI-MRI scanner," IEEE Trans. Med. Imag., vol. 35, no. 9, pp. 1993-2004, Sep. 2016. [Online]. Available: http://doi.org/10.1109/TMI.2016.2542041

[15] M. Graeser, T. Knopp, M. Grüttner, T. F. Sattel, and T. M. Buzug, "Analog receive signal processing for magnetic particle imaging," Med. Phys., vol. 40, no. 4, p. 042303, Apr. 2013. [Online]. Available: http://doi.org/10.1118/1.4794482

[16] P. W. Goodwill, K. Lu, B. Zheng, and S. M. Conolly, "An X-space magnetic particle imaging scanner," Rev. Sci. Instrum., vol. 83 no. 3, p. 033708, Mar. 2012. [Online]. Available: http://doi.org/10. $1063 / 1.3694534$

[17] V. Schulz, M. Straub, M. Mahlke, S. Hubertus, T. Lammers, and F. Kiessling, "A field cancellation signal extraction method for magnetic particle imaging," IEEE Trans. Magn., vol. 51, no. 2, pp. 1-4, Feb. 2015. [Online]. Available: http://doi.org/10.1109/TMAG.2014. 2325852

[18] A. von Gladiss, M. Graeser, P. Szwargulski, T. Knopp, and T. M. Buzug, "Hybrid system calibration for multidimensional magnetic particle imaging," Phys. Med. Biol., vol. 62, no. 9, pp. 3392-3406, Apr. 2017. [Online]. Available: http://doi.org/10.1088/1361-6560/ aa5340

[19] J. Rahmer, "Analysis of a 3-D system function measured for magnetic particle imaging," IEEE Trans. Med. Imag., vol. 31, no. 6 , pp. 1289-1299, Jan. 2012. [Online]. Available: http://doi.org/10.1109/TMI.2012.2188639

[20] J. Rahmer et al., "Signal encoding in magnetic particle imaging: Properties of the system function," BMC Med. Imag., vol. 9 p. 4, Dec. 2009. [Online]. Available: http://doi.org/10.1186/14712342-9-4

[21] T. Knopp et al., "2D model-based reconstruction for magnetic particle imaging," Med. Phys., vol. 37, no. 2, pp. 485-491, 2010. [Online]. Available: http://doi.org/10.1118/1.3271258
[22] M. Straub, T. Lammers, F. Kiessling, and V. Schulz, "Flexible and modular MPI simulation framework and its use in modeling a $\mathrm{MPI}$," IEEE Trans. Magn., vol. 51, no. 2, Feb. 2015, Art. no. 650204. [Online]. Available: http://doi.org/10.1109/TMAG.2014.2329733

[23] T. Knopp, S. Biederer, T. Sattel, and T. M. Buzug, "Singular value analysis for magnetic particle imaging," in Proc. IEEE Nucl. Sci. Symp. Conf. Rec., Oct. 2008, pp. 4525-4529. [Online]. Available: http://doi.org/10.1109/NSSMIC.2008.4774296

[24] T. Knopp et al., "Weighted iterative reconstruction for magnetic particle imaging," Phys. Med. Biol., vol. 55, no. 6, pp. 1577-1589, 2010 [Online]. Available: http://doi.org/10.1088/0031-9155/55/6/003

[25] A. Dax, "On row relaxation methods for large constrained least squares problems," SIAM J. Sci. Comput., vol. 14, no. 3, pp. 570-584, May 1993. [Online]. Available: http://doi.org/10.1137/0914036

[26] A. Halkola, T. M. Buzug, J. Rahmer, B. Gleich, and C. Bontus, System Calibration Unit for Magnetic Particle Imaging: Focus Field Based System Function. Berlin, Germany: Springer, 2012, pp. 27-31. [Online] Available: http://doi.org/10.1007/978-3-642-24133-8_5

[27] R. H. Byrd, P. Lu, J. Nocedal, and C. Zhu, "A limited memory algorithm for bound constrained optimization," SIAM J. Sci. Comput., vol. 16, no. 5, pp. 1190-1208, 1995. [Online]. Available: http://doi.org/10.1137/0916069

[28] C. Zhu, R. H. Byrd, P. Lu, and J. Nocedal, "Algorithm 778: L-BFGS-B: Fortran subroutines for large-scale bound-constrained optimization," ACM Trans. Math. Softw., vol. 23, no. 4, pp. 550-560, 1997. [Online]. Available: http://doi.org/10.1145/279232.279236

[29] M. Storath et al., "Edge preserving and noise reducing reconstruction for magnetic particle imaging," IEEE Trans. Med. Imag., vol. 36, no. 1, pp. 74-85, Jan. 2017. [Online]. Available: http://doi.org/10. 1109/TMI.2016.2593954

[30] B. Gleich, J. Weizenecker, and J. Borgert, "Experimental results on fast 2D-encoded magnetic particle imaging," Phys. Med. Biol., vol. 53, no. 6, p. N81-4, Feb. 2008. [Online]. Available: http://doi.org/10.1088/00319155/53/6/N01

[31] M. G. Kaul et al., "Combined preclinical magnetic particle imaging and magnetic resonance imaging: Initial results in mice," RöFoFortschritte auf dem Gebiet der Röntgenstrahlen und der bildgebenden Verfahren, vol. 187, no. 5, pp. 347-352, Apr. 2015. [Online]. Available: http://doi.org/10.1055/s-0034-1399344

[32] D. Ho, X. Zhao, S. Gao, C. Hong, D. E. Vatner, and S. F. Vatner, Heart Rate and Electrocardiography Monitoring in Mice, vol. 1. Hoboken, NJ, USA: Wiley, Mar. 2011, pp. 123-139. [Online]. Available: http://doi.org/10.1002/9780470942390.mo100159 